\title{
Basic Minimal Dominating Functions of Quadratic Residue Cayley Graphs
}

\author{
Jeelani Begum, $\mathbf{S}^{1 *}$ and Maheswari, $\mathbf{B}^{2}$ \\ ${ }^{1}$ Department of Mathematics, Madanapalle Institute of Technology \& Science, Madanapalle, \\ Andhra Pradesh, India (*sjb.maths@gmail.com) \\ ${ }^{2}$ Department of Applied Mathematics, Sri Padmavati Mahila Visvavidyalayam, Tirupati, Andhra \\ Pradesh, India (maherahul.55@gmail.com)
}

\begin{abstract}
Domination arises in the study of numerous facility location problems where the number of facilities is fixed and one attempt to minimize the number of facilities necessary so that everyone is serviced. This problem reduces to finding a minimum dominating set in the graph corresponding to this network. In this paper we study the minimal dominating functions and basic minimal dominating functions of quadratic residue Cayley graphs and results on these functions have been obtained.
\end{abstract}

Key words: Quadratic Residue Cayley Graph, Minimal Dominating Functions, Basic Minimal Dominating Functions.

\section{[AMS (MOS) Subject Classification: 05C69]}

\section{INTRODUCTION}

Domination theory of graphs is an important branch of Graph Theory and has many applications in Engineering, Communication Networks and many others. Allan and Laskar (1978); Allan et al. (1984); Cockayne and Hedetniemi (1977); Haynes et al. (1998) have studied various domination parameters of graphs. Graphs associated with certain arithmetical functions which are usually called arithmetical graphs have been studied extensively by many researchers. Here we consider quadratic residue Cayley graph $\mathrm{G}\left(\mathrm{Z}_{\mathrm{p}}, \mathrm{Q}\right)$. First we derive results on minimal dominating functions of $G\left(Z_{p}, Q\right)$ and prove that these functions are basic minimal dominating functions in certain cases. We start with the definition of a Quadratic Residue Cayley graph.

\section{QUADRATIC RESIDUE CAYLEY GRAPH AND ITS PROPERTIES}

Definition 2.1: Let $p$ be an odd prime and $n$, a positive integer such that $n \neq \equiv 0(\bmod p)$. If the

quadratic congruence, $\mathrm{x}^{2} \equiv \mathrm{n}(\bmod \mathrm{p})$ has a solution then $\mathrm{n}$ is called a quadratic residue modulo pand it is written as $\mathrm{nRp}$.

If the congruence $x^{2} \equiv n(\bmod p)$ has no solution, then $n$ is called the quadratic non-residue $\bmod p$ and is written as $n \bar{R} p$. 
Example: For the prime 11, we have

$$
\begin{aligned}
& 1^{2} \equiv 1, \quad 2^{2} \equiv 4, \quad 3^{2} \equiv 9, \quad 4^{2} \equiv 5, \quad 5^{2} \equiv 3(\bmod 11) \text { also } \\
& 6^{2} \equiv 3, \quad 7^{2} \equiv 5, \quad 8^{2} \equiv 9, \quad 9^{2} \equiv 4, \quad 10^{2} \equiv 1(\bmod 11) .
\end{aligned}
$$

Consequently the quadratic residues mod 11 are 1, 3, 4, 5, 9 and non-residues are 2, 6, 7, 8, 10 .

Definition 2.2: Let $\mathrm{p}$ be an odd prime, $\mathrm{S}$, the set of quadratic residues modulo pand let $S^{*}=\{s, p-s / s \in S, s \neq p\}$. The quadratic residue Cayley graph $G\left(Z_{p}, Q\right)$ is defined as the graph whose vertex set is $Z_{p}=\{0,1,2,3, \ldots \ldots,(p-1)\}$ and the edge set

$$
\mathrm{E}=\left\{(\mathrm{x}, \mathrm{y}) / \mathrm{x}-\mathrm{y} \text { or } \mathrm{y}-\mathrm{x} \text { is in } \mathrm{S}^{*}\right\} .
$$

The graph $\mathrm{G}\left(\mathrm{Z}_{13}, \mathrm{Q}\right)$ is given below (Fig 1)..

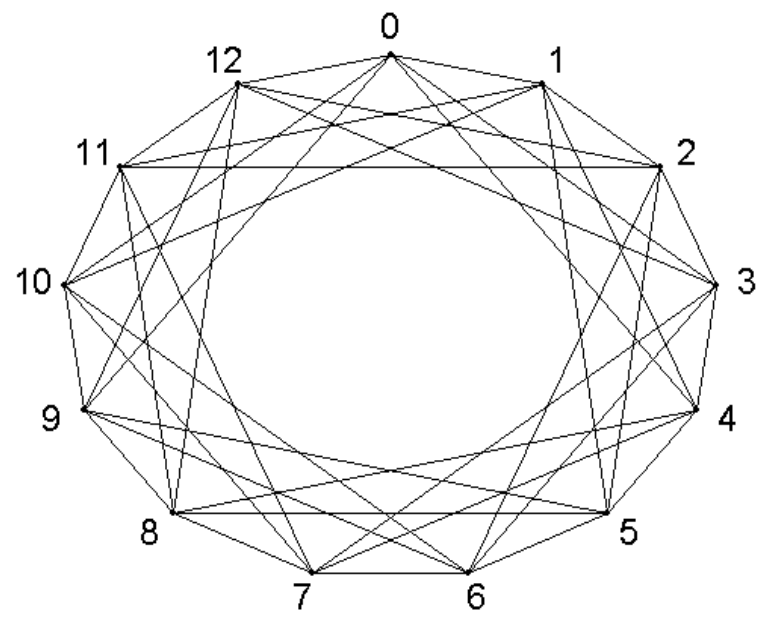

Figure 1. $\mathrm{G}\left(\mathrm{Z}_{13}, \mathrm{Q}\right)$.

We make use of the following results whose proofs can be found in Madhavi and Maheswari (2011) and Maheswari and Madhavi (2009).

Lemma 2.3: The graph $G\left(Z_{p}, Q\right)$ is $\left|S^{*}\right|$ - regular and the number of edges of $G\left(Z_{p}, Q\right)$ is $\frac{\left|Z_{p}\right|\left|S^{*}\right|}{2}$.

Theorem 2.4: The graph $\mathrm{G}\left(\mathrm{Z}_{\mathrm{p}}, \mathrm{Q}\right)$ is complete if and only if, $\mathrm{p} X\left(a^{2}+b^{2}\right)$, for any positive integers $\mathrm{a}$ and $\mathrm{b}$.

Theorem 2.5: The graph $G\left(Z_{p}, Q\right)$ is complete if $p$ is of the form $4 m+3$.

Theorem 2.6: If $\mathrm{p}$ is of the form $4 \mathrm{~m}+1$, then the sets $\mathrm{Q}$ and $\mathrm{S}^{*}$ are the same, so that the graph $\mathrm{G}$ $\left(\mathrm{Z}_{\mathrm{p}}, \mathrm{Q}\right)$ is not complete. 


\section{BASIC MINIMAL DOMINATING FUNCTIONS}

Definition 3.1: A function $f: V \rightarrow[0,1]$ is called a dominating function (DF) of $\mathrm{G}$ if $f(\mathrm{~N}[\mathrm{v}])=$ $\sum_{\mathrm{u} \in N[v]} f(u) \geq 1$, for each $\mathrm{v} \in \mathrm{V}$.

Here $N[v]$ is the neighborhood set of $v$ defined by $N[v]=\{x \in V /(x, v) \in E\} \cup\{v\}$.

Definition 3.2: Let $f$ and $g$ be functions from $V \rightarrow[0,1]$. We define $\mathrm{f}<\mathrm{g}$, if $\mathrm{f}(\mathrm{u}) \leq \mathrm{g}(\mathrm{u})$, for all $\mathrm{u} \in \mathrm{V}$, with strict inequality for at least one vertex $\mathrm{u}$. A DF $\mathrm{g}$ of $\mathrm{G}$ is called a minimal dominating function (MDF) if for all $\mathrm{f}<\mathrm{g}$, fis not a dominating function.

Definition 3.3: A MDF $f$ of a graph is called basic minimal dominating function (BMDF)if $f$ cannot be expressed as a proper convex combination of two distinct MDFs.

Definition 3.4: Let $f$ be a $D F$ of a graph $G(V, E)$. The boundary set of $f$ is defined by

$$
B_{f}=\left\{u \in V / f(N[u])=\sum_{x \in N[u]} f(x)=1\right\} .
$$

The positive set of $\mathrm{f}$ is defined by

$$
P_{f}=u \in V / f(u)>0 \text {. }
$$

We state the following theorems which are useful for obtaining subsequent results whose proofs can be found in Arumugam and Reji Kumar (2008).

Theorem 3.5: Let $\mathrm{f}$ be a MDF of a graph $\mathrm{G}(\mathrm{V}, \mathrm{E})$ with $B_{f}=\left\{\mathrm{v}_{1}, \mathrm{v}_{2}, \ldots ., \mathrm{v}_{\mathrm{m}}\right\}$ and $P_{f}^{\prime}=\{\mathrm{u} \in \mathrm{V} / 0<\mathrm{f}(\mathrm{u})<1\}=\left\{\mathrm{u}_{1}, \mathrm{u}_{2}, \ldots \ldots, \mathrm{u}_{\mathrm{n}}\right\}$. Let $\mathrm{A}=\left(\mathrm{a}_{\mathrm{ij}}\right)$ be an $\mathrm{m} \times \mathrm{n}$ matrix defined by

$$
a_{i j}=\left\{\begin{array}{l}
1, \text { if } v_{i} \text { is adjacent to } u_{j} \text { or } v_{i}=u_{j} \\
0, \text { otherwise. }
\end{array}\right.
$$

Consider the system of linear equations given by

$$
\sum_{j=1}^{n} a_{i j} x_{j}=0,(1 \leq i \leq m) \ldots \ldots \ldots \ldots \ldots \ldots \ldots . . .\left(S_{1}\right)
$$

Then $\mathrm{f}$ is a BMDF if and only if $\left(\mathrm{S}_{1}\right)$ does not have a non-trivial solution.

Corollary 3.6: Let $\mathrm{G}(\mathrm{V}, \mathrm{E})$ be a graph without isolated vertices. Let $\mathrm{S}$ be a MDS of G.

Then $f=\chi_{s}$ is a BMDF.

We now derive some results related to MDFs and BMDFs of $G\left(Z_{p}, Q\right)$. 


\section{RESULTS}

Theorem 4.1: A function $f: V \rightarrow[0,1]$ defined by

$$
f(v)=\frac{1}{q}, \forall \mathrm{v} \in \mathrm{V}, \mathrm{q}>0,
$$

becomes a DF of $G\left(Z_{p}, Q\right)$. It is a MDF if $q=p$, otherwise not a MDF.

Proof: Suppose $p=4 m+3$. Then $G\left(Z_{p}, Q\right)$ is complete.

The neighbourhood $\mathrm{N}[\mathrm{v}]$ of $\mathrm{v}$ in $\mathrm{V}$ consists of $\mathrm{p}$ vertices.

Let $f: V \rightarrow[0,1]$ be defined by

$$
f(v)=\frac{1}{q}, \forall \mathrm{v} \in \mathrm{V} .
$$

Case 1: Suppose $q=p$.

Then $f(v)=\frac{1}{p}, \forall \mathrm{v} \in \mathrm{V}$.

And $\sum_{u \in N[v]} f(u)=\underbrace{\frac{1}{p}+\frac{1}{p}+\ldots+\frac{1}{p}}_{p \text {-times }}=\frac{p}{p}=1, \forall \mathrm{v} \in \mathrm{V}$.

Therefore $\mathrm{f}$ is a DF.

We now check for the minimality of $f$.

Suppose $g: V \rightarrow[0,1]$ is a function defined by

$$
g(v)= \begin{cases}r, & \text { if } v=v_{k}, \\ \frac{1}{p}, & \text { if } v \in V-\left\{v_{k}\right\} .\end{cases}
$$

where $\mathrm{r}<1 / \mathrm{p}$ and $\mathrm{v}_{\mathrm{k}} \in \mathrm{V}$.

$$
\text { Then } \begin{aligned}
\sum_{u \in N[v]} g(u) & =\underbrace{\frac{1}{p}+\frac{1}{p}+\ldots+\frac{1}{p}}_{(p-1) \text {-times }}+r \\
<\frac{(p-1)}{p} & +\frac{1}{p} \\
& =\frac{p}{p}=1 .
\end{aligned}
$$

Therefore $\sum_{u \in N[v]} g(u) \geq 1, \forall \mathrm{v} \in \mathrm{V}$.

(C) CNCS, Mekelle University 
So $\mathrm{g}$ is not a DF.

Since $g$ is defined arbitrarily, it follows that there exists no $g<f$ such that $g$ is a DF.

Thus $\mathrm{f}$ is a MDF.

Case 2: Suppose $0<\mathrm{q}<\mathrm{p}$.

Then $\sum_{u \in N[v]} f(u)=\underbrace{\frac{1}{q}+\frac{1}{q}+\ldots . .+\frac{1}{q}}_{p \text {-times }}=\frac{p}{q}$.

Since $\mathrm{q}<\mathrm{p}$, it follows that $\frac{p}{q}>1$.

Thus $\sum_{u \in N[v]} f(u)>1, \quad \forall v \in V$.

Therefore $\mathrm{f}$ is a DF.

To prove that $\mathrm{f}$ is minimal.

Define $g: V \rightarrow[0,1]$ by

$$
g(v)= \begin{cases}r, & \text { if } v=v_{k}, \\ \frac{1}{q}, & \text { if } v \in V-\left\{v_{k}\right\} .\end{cases}
$$

where $0<\mathrm{r}<\frac{1}{q}$ and $\mathrm{v}_{\mathrm{k}} \in \mathrm{V}$.

Since strict inequality holds at the vertex $v_{k}$ of $V$, it follows that $g<f$.

Then $\sum_{u N[v]} g(u)=r+\underbrace{\frac{1}{q}+\frac{1}{q}+\ldots . .+\frac{1}{q}}_{(p-1) \text {-times }}$

$<\frac{1}{q}+\frac{p-1}{q}=\frac{p}{q}$

But $\frac{p}{q}>1$. It follows that $\sum_{u \in N[v]} g(u) \geq 1, \quad \forall v \in V$.

Thus $\mathrm{g}$ is a DF.

This implies that $\mathrm{f}$ is not a MDF.

Theorem 4.2: A function $f: V \rightarrow[0,1]$ defined by $f(v)=\frac{1}{q}, \forall \mathrm{v} \in \mathrm{V}$, 
becomes a $\mathrm{DF}$ of $\mathrm{G}\left(\mathrm{Z}_{\mathrm{p}}, \mathrm{Q}\right)$. It becomes MDF if $\mathrm{q}=\left|S^{*}\right|+1$. Otherwise it is not minimal.

Proof: Suppose $\mathrm{p}=4 \mathrm{~m}+1$. Consider $\mathrm{G}\left(\mathrm{Z}_{\mathrm{p}}, \mathrm{Q}\right)$ with vertex set $\mathrm{V}=\{0,1,2, \ldots \ldots \ldots(\mathrm{p}-1)\}$.

Then every neighbourhood $\mathrm{N}[\mathrm{v}]$ of $\mathrm{v}$ in $\mathrm{V}$ consists of $\left|S^{*}\right|+1$ vertices.

Let $\left|S^{*}\right|+1=\mathrm{m}$.

Let $\mathrm{f}$ be a function defined as in the hypothesis.

Case 1: Suppose $\mathrm{q}=\mathrm{m}$.

Then $f(v)=\frac{1}{m}, \forall \mathrm{v} \in \mathrm{V}$.

And $\sum_{u \in N[v]} f(u)=\underbrace{\frac{1}{m}+\frac{1}{m}+\ldots+\frac{1}{m}}_{m \text {-times }}=\frac{m}{m}=1, \forall \mathrm{v} \in \mathrm{V}$.

Therefore $\mathrm{f}$ is a DF.

We now check for the minimality of $f$.

Suppose $g: V \rightarrow[0,1]$ is a function defined by

$$
g(v)= \begin{cases}r, & \text { if } v=v_{k}, \\ \frac{1}{m}, & \text { if } v \in V-\left\{v_{k}\right\} .\end{cases}
$$

where $\mathrm{r}<1 / \mathrm{m}$ and $\mathrm{v}_{\mathrm{k}} \in \mathrm{V}$.

If $\mathrm{v}_{\mathrm{k}} \in \mathrm{N}[\mathrm{v}]$ then

$$
\begin{aligned}
& \sum_{u \in N[v]} g(u)=\underbrace{\frac{1}{m}+\frac{1}{m}+\ldots+\frac{1}{m}}_{(m-1)-\text { times }}+r \\
&<\frac{(m-1)}{m}+\frac{1}{m}=\frac{m}{m}=1 .
\end{aligned}
$$

If $\mathrm{v}_{\mathrm{k}} \notin \mathrm{N}[\mathrm{v}]$ then

$$
\begin{aligned}
\sum_{u \in N[v]} g(u) & =\underbrace{\frac{1}{m}+\frac{1}{m}+\ldots+\frac{1}{m}}_{m \text {-times }} \\
& =\frac{m}{m}=1 .
\end{aligned}
$$

Therefore $\sum_{u \in N[v]} g(u) \geq 1, \forall \mathrm{v} \in \mathrm{V}$. 
So $g$ is not a DF.

Since $g$ is defined arbitrarily, it follows that there exists no $g<f$ such that $g$ is a DF.

Thus $f$ is a MDF.

Case 2: Suppose $0<\mathrm{q}<\mathrm{m}$.

Then $\sum_{u \in N[v]} f(u)=\underbrace{\frac{1}{q}+\frac{1}{q}+\ldots . .+\frac{1}{q}}_{m \text {-times }}$

$$
=\frac{m}{q} .
$$

Since $\mathrm{q}<\mathrm{m}$, it follows that $\frac{m}{q}>1$.

Thus $\sum_{u \in N[v]} f(u) \geq 1, \quad \forall v \in V$.

Therefore $\mathrm{f}$ is a DF.

We now check for the minimality of $f$.

Define $g: V \rightarrow[0,1]$ by

$$
g(v)= \begin{cases}r, & \text { if } v=v_{k}, \\ \frac{1}{q}, & \text { if } v \in V-\left\{v_{k}\right\} .\end{cases}
$$

where $0<\mathrm{r}<\frac{1}{q}$ and $\mathrm{v}_{\mathrm{k}} \in \mathrm{V}$.

Since strict inequality holds at the vertex $\mathrm{v}_{\mathrm{k}}$ of $\mathrm{V}$, it follows that $\mathrm{g}<\mathrm{f}$.

If $\mathrm{v}_{\mathrm{k}} \in \mathrm{N}[\mathrm{v}]$ then

$$
\begin{aligned}
& \sum_{u \in N[v]} g(u)=\underbrace{\frac{1}{m}+\frac{1}{m}+\ldots+\frac{1}{m}}_{(m-1) \text {-times }}+r \\
& <\underbrace{\frac{1}{q}+\frac{1}{q}+\ldots . .+\frac{1}{q}}_{m \text {-times }}=\frac{m}{q} .
\end{aligned}
$$

But $\frac{m}{q}>1$.

If $\mathrm{v}_{\mathrm{k}} \notin \mathrm{N}[\mathrm{v}]$ then

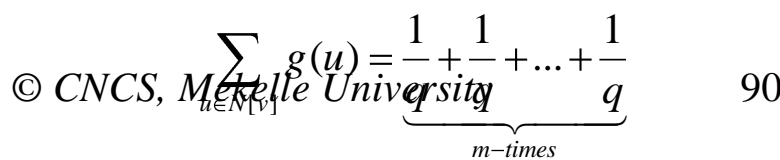




$$
=\frac{m}{q}>1
$$

It follows that $\sum_{u \in N(v)} g(u) \geq 1, \forall v \in V$.

This implies that $\mathrm{g}$ is a DF. So $\mathrm{f}$ is not a MDF in this case.

Theorem 4.3: Let $f: V \rightarrow[0,1]$ be a function defined by

$f(v)=\frac{1}{(r+1)}, \forall \mathrm{v} \in \mathrm{V}$, where $\mathrm{r}$ denotes the degree of $\mathrm{v}$ in $\mathrm{V}$.

Then

(i) $f$ is not a BMDF for $p=4 m+3$.

(ii) $\mathrm{f}$ is a BMDF for $\mathrm{p}=4 \mathrm{~m}+1$.

Proof: Consider the graph $\mathrm{G}\left(\mathrm{Z}_{\mathrm{p}}, \mathrm{Q}\right)$.

Case 1 : Suppose $\mathrm{p}=4 \mathrm{~m}+3$.

Then $\mathrm{G}\left(\mathrm{Z}_{\mathrm{p}}, \mathrm{Q}\right)$ is complete and $(\mathrm{p}-1)$ - regular. i.e., every vertex $\mathrm{v}$ in $\mathrm{V}$ is adjacent to ( $\left.\mathrm{p}-1\right)$ vertices. Then $r=p-1$. Let $\mathrm{f}$ be the function defined as in the hypothesis.

By Theorem 4.1 Case $1, \mathrm{f}$ is a MDF.

We claim that $\mathrm{f}$ is not a BMDF.

Here $f(v)=\frac{1}{(p-1+1)}, \quad$ since $r=p-1$

$$
=\frac{1}{p}<1, \forall \mathrm{v} \in \mathrm{V} \text {. }
$$

Therefore

$$
\begin{aligned}
\sum_{x \in N[u]} f(x)=\underbrace{\frac{1}{p}+\frac{1}{p}+\ldots \ldots \ldots+\frac{1}{p}}_{(p-\text { times })} & \\
& =\mathrm{p} \times \frac{1}{p}=1, \forall \mathrm{v} \in \mathrm{V} .
\end{aligned}
$$

Since $\mathrm{u}$ is arbitrary it follows that $\sum_{x \in N[u]} f(x)=1, \forall u \in V$.

As there are $\mathrm{p}$ vertices in $\mathrm{V}$ we get 


$$
\begin{aligned}
B_{f} & =\left\{u \in V / f(N[u])=\sum_{x \in N[u]} f(x)=1\right\} \\
& =\left\{\mathrm{v}_{1}, \mathrm{v}_{2}, \mathrm{v}_{3}, \ldots, \mathrm{v}_{\mathrm{p}}\right\} \text { say. }
\end{aligned}
$$

And $P_{f}^{\prime}=u \in V / 0<f(u)<1=\left\{\mathrm{u}_{1}, \mathrm{u}_{2}, \mathrm{u}_{3}, \ldots \mathrm{u}_{\mathrm{p}}\right\}$ say.

That is $\mathrm{f}$ is a MDF with $B_{f}=P_{f}^{\prime}=V$.

Let $\mathrm{A}=\left(\mathrm{a}_{\mathrm{ij}}\right)$ be $\mathrm{a} \mathrm{p} \times \mathrm{p}$ matrix defined by

$$
\begin{aligned}
\mathrm{a}_{\mathrm{ij}} & =1, \text { if } \mathrm{v}_{\mathrm{i}} \text { is adjacent to } u_{j} \text { or } v_{i}=u_{j}, \\
& =0 \text {, otherwise. }
\end{aligned}
$$

Then the system of linear equations associated with $\mathrm{f}$ is defined by

$$
\sum_{j=1}^{p} a_{i j} x_{j}=0 \text { where } \mathrm{i}=1,2,3, \ldots, \mathrm{p} .
$$

Since every vertex $\mathrm{v}$ in $B_{f}$ is adjacent to all the $\mathrm{p}$ vertices of $\mathrm{V}$ we have $\mathrm{a}_{\mathrm{ij}}=1$, where $1 \leq \mathrm{i} \leq \mathrm{p}, 1 \leq \mathrm{j} \leq \mathrm{p}$.

That is

$$
\begin{array}{lccc}
\text { For } v_{1} \in B_{f}, & 1 . x_{1}+1 . x_{2}+\ldots+1 . x_{p}=0 \\
\text { For } v_{2} \in B_{f}, & 1 . x_{1}+1 . x_{2}+\ldots+1 . x_{p}=0 \\
\ldots & \ldots & \ldots & \ldots \\
\ldots & \ldots & \ldots & \ldots \\
{\text { For } v_{p} \in B_{f},}_{1 . x_{1}+1 . x_{2}+\ldots+1 . x_{p}=0 .}
\end{array}
$$

This implies $x_{1}+x_{2}+\ldots \ldots+x_{p}=0$, which has a non-trivial solution.

By Theorem 3.5, it follows that $\mathrm{f}$ is not a BMDF.

Case 2: Suppose $\mathrm{p}=4 \mathrm{~m}+1$.

Then $\mathrm{G}\left(\mathrm{Z}_{\mathrm{p}}, \mathrm{Q}\right)$ is a regular graph and it is $\left|S^{*}\right|$ regular.

That is $\mathrm{r}=\left|S^{*}\right|$. Let $\mathrm{f}$ be the function defined as in the hypothesis.

By Theorem 4.2 Case 1, $\mathrm{f}$ is a MDF.

We claim that $\mathrm{f}$ is a BMDF.

Here $f(v)=\frac{1}{\left(\left|S^{*}\right|+1\right)}<1, \forall \mathrm{v} \in \mathrm{V}$. 
Then $\sum_{u \in N[v]} f(u)=\underbrace{\frac{1}{(r+1)}+\frac{1}{(r+1)}+\ldots \ldots \ldots \ldots \ldots+\frac{1}{(r+1)}}_{(r+1) \text {-times }}=1, \forall \mathrm{v} \in \mathrm{V}$,

since each $\mathrm{N}[\mathrm{v}]$ consists of $\mathrm{r}+1$ vertices.

Therefore $B_{f}=\left\{u \in V / f(N[u])=\sum_{x \in N[u]} f(x)=1\right\}=\left\{\mathrm{v}_{1}, \mathrm{v}_{2}, \mathrm{v}_{3}, \ldots \mathrm{v}_{\mathrm{p}}\right\}$ ( as in Case 1)

Similarly $P_{f}^{\prime}=u \in V / 0<f(u)<1=\left\{\mathrm{u}_{1}, \mathrm{u}_{2}, \mathrm{u}_{3}, \ldots, \mathrm{u}_{\mathrm{p}}\right\}$ say.

Let $\mathrm{A}=\left(\mathrm{a}_{\mathrm{ij}}\right)$ be $\mathrm{a} \mathrm{p} \times \mathrm{p}$ matrix defined by

$$
\begin{aligned}
\mathrm{a}_{\mathrm{ij}} & =1 \text {, if } \mathrm{v}_{\mathrm{i}} \text { is adjacent to } u_{j} \text { or } v_{i}=u_{j}, \\
& =0 \text {, otherwise. }
\end{aligned}
$$

Then the system of linear equations associated with $\mathrm{f}$ is defined by

$$
\sum_{j=1}^{p} a_{i j} x_{j}=0 \text { where } \mathrm{i}=1,2,3, \ldots, \mathrm{p} .
$$

Since every vertex $\mathrm{v}$ in $B_{f}, \mathrm{~N}[\mathrm{v}]$ consists of $(\mathrm{r}+1)$ vertices of $\mathrm{V}$ we have $\mathrm{a}_{\mathrm{ij}}=1$ for $\mathrm{r}+1$ variables where $1 \leq \mathrm{i} \leq \mathrm{p}, 1 \leq \mathrm{j} \leq \mathrm{p}$.

That is

$$
\begin{aligned}
& \text { For } v_{1} \in B_{f}, 1 . x_{1}+1 . x_{2}+\underbrace{0+\ldots+0}_{(p-(r+1))-\text { times }}+1 . x_{p}=0 \\
& \text { For } v_{2} \in B_{f}, 1 . x_{2}+1 . x_{3}+1 \cdot x_{4}+\underbrace{0+\ldots+0}_{(p-(r+1))-\text { times }}=0 \\
& \begin{array}{llll}
\cdots & \cdots & \cdots & \cdots
\end{array} \\
& \begin{array}{llll}
\cdots & \ldots & \ldots & \ldots
\end{array} \\
& \text { For } v_{p} \in B_{f}, 1 . x_{p}+1 . x_{p-1}+\underbrace{0+\ldots+0}_{(p-(r+1))-\text { times }}+1 . x_{1}=0 \text {. }
\end{aligned}
$$

As the above system of equations has a trivial solution, by Theorem 3.5, it follows that $\mathrm{f}$ is a BMDF.

For example, consider the graph $\mathrm{G}\left(\mathrm{Z}_{5}, \mathrm{Q}\right)$.

The graph of $\mathrm{G}\left(\mathrm{Z}_{5}, \mathrm{Q}\right)$ is given below.

Since it is 2-regular, $\left|S^{*}\right|=r=2<5$.

Define a function $f: V \rightarrow[0,1]$ by

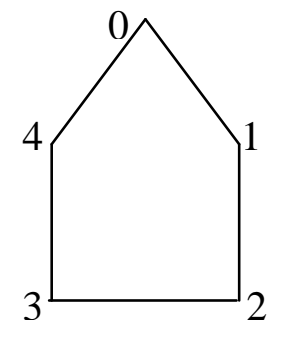

$\mathbf{G}\left(\mathbf{Z}_{5}, \mathbf{Q}\right)$ 


$$
f(v)=\frac{1}{r+1}=\frac{1}{3}, \forall \mathrm{v} \in \mathrm{V} .
$$

Then

$$
\begin{array}{lcccccc}
v: & 0 & 1 & 2 & 3 & 4 & \\
f(v): & \frac{1}{3} & \frac{1}{3} & \frac{1}{3} & \frac{1}{3} & \frac{1}{3} & \\
\sum_{u \in N[v]} f(u): & 1 & 1 & 1 & 1 & 1
\end{array}
$$

Clearly $\mathrm{f}$ is a MDF.

Here $B_{f}=\{0,1,2,3,4\}=\mathrm{V}=P_{f}^{\prime}$.

The system of linear equations is given by

$$
\begin{aligned}
& \text { For } v_{1}=0 \in B_{f}, x_{1}+x_{2}+x_{5}=0 \ldots \ldots .(1) \\
& \text { For } v_{2}=1 \in B_{f}, x_{1}+x_{2}+x_{3}=0 \ldots \ldots .(2) \\
& \text { For } v_{3}=2 \in B_{f}, x_{2}+x_{3}+x_{4}=0 \ldots \ldots .(3) \\
& \text { For } v_{4}=3 \in B_{f}, x_{3}+x_{4}+x_{5}=0 \ldots \ldots .(4) \\
& \text { For } v_{5}=4 \in B_{f}, x_{1}+x_{4}+x_{5}=0 \ldots \ldots .(5)
\end{aligned}
$$

From (1) \& (2) we have $\mathrm{x}_{3}=\mathrm{x}_{5}$

From (2) \& (3) we have $\mathrm{x}_{1}=\mathrm{x}_{4} \ldots \ldots \ldots \ldots \ldots . .(7)$

From (3) \& (4) we have $\mathrm{x}_{2}=\mathrm{x}_{5}$

From (4) \& (5) we have $x_{1}=x_{3}$.

Therefore $\mathrm{x}_{1}=\mathrm{x}_{2}=\mathrm{x}_{3}=\mathrm{x}_{4}=\mathrm{x}_{5}=0$.

This implies a trivial solution. Hence $\mathrm{f}$ is a BMDF.

Lemma 4.4: In $\mathrm{G}\left(\mathrm{Z}_{\mathrm{p}}, \mathrm{Q}\right)$, the function $f=\chi_{D}$ is a BMDF, where $\mathrm{D}$ is a MDS of $\mathrm{G}\left(\mathrm{Z}_{\mathrm{p}}, \mathrm{Q}\right)$.

Proof: Let $f=\chi_{D}$, where $\mathrm{D}$ is a MDS of $\mathrm{G}\left(\mathrm{Z}_{\mathrm{p}}, \mathrm{Q}\right)$. Since $\mathrm{f}$ assumes only the values 0 and 1 , we have $P_{f}^{\prime}=u \in V / 0<f(u)<1=\phi$ and by Corollary 3.6, it follows that $\mathrm{f}$ is a BMDF.

Theorem 4.5: The number of BMDFs of $G\left(Z_{p}, Q\right)=p$, when $p=4 m+3$

$$
=\mathrm{k} \text {, when } \mathrm{p}=4 \mathrm{~m}+1
$$

where $\mathrm{k}$ is the number of MDSs of $\mathrm{G}\left(\mathrm{Z}_{\mathrm{p}}, \mathrm{Q}\right)$. 
Proof: Case 1: Suppose $p=4 m+3$. Then $G\left(Z_{p}, Q\right)$ is complete and hence each vertex forms a MDS. By Theorem 4 of Jeelani Begum and Maheswari (2011), the number of MDSs is p. By Lemma 4.4, we have $\mathrm{p}$ functions which are BMDFs. Therefore there are $\mathrm{p}$ BMDFs of $\mathrm{G}\left(\mathrm{Z}_{\mathrm{p}}, \mathrm{Q}\right)$.

Case 2: Suppose $p=4 m+1$. Then by the Algorithm presented in Jeelani Begum\& Maheswari (2011), the total number of MDSs of $G\left(Z_{p}, Q\right)$ is $k$. Thus we have k such functions which become BMDFs of $\mathrm{G}\left(\mathrm{Z}_{\mathrm{p}}, \mathrm{Q}\right)$.

\section{CONCLUSION}

In this paper we derived the results related to minimal dominating functions and basic minimal dominating functions of quadratic residue Cayley graphs in certain cases. Further we discuss about the possible number of basic minimal dominating functions in those cases.

\section{REFERENCES}

Allan, R. B \& Laskar, R. 1978. On domination and independent domination number of a graph , Discrete Math., 23: 73-76.

Allan, R. B., Lasker, R \& Hedetniemi, S.T. 1984. A note on total domination. Discrete Math., 49:713.

Arumugam, S \& Reji Kumar, K. 2008. Basic Minimal Dominating Functions, Utilitas Math., 77: 235-247.

Cockayne, E.J \& Hedetniemi, S.T. 1977. Towards a theory of domination in graphs, Networks, 7: $247-261$.

Haynes, T.W., Hedetniemi, S.T \& Slater, J.S. 1998. Fundamentals of Domination in Graphs, Marcel Decker, Inc.

Jeelani Begum, S \& Maheswari, B. 2011. Minimal dominating functions of quadratic residue cayley graphs, Proceedings of International Conference on Advances in Mathematical \& Computational Methods (AMCM-2011), SPMVV, Tirupati, A.P., India, 2: 329-332.

Madhavi, L \& Maheswari,B. 2011. Edge Cover Domination in Mangoldt Graph, Momona Ethiopian Journal of Science, 3(1):37-51.

Maheswari, B\& Madhavi Lavaku. 2009. Enumeration of Triangles andHamilton Cycles in QuadraticResidue Cayley Graphs. Chamchuri J. Math., 1(1): 95-103. 